\title{
Out of Hospital Cardiac Arrest: Saudi Red Crescent Experience Throughout COVID-19 Era
}

\author{
Yousef M Alsofayan (iD) \\ Saqer M Althunayyan (D) ${ }^{2}$ \\ Mohamed A Mohamed (iD) \\ Saud H Alhabeeb (D) \\ Mohammad I Altuwaijri (D) ' \\ Fahad S Alhajjaj (D) ${ }^{3}$ \\ Jalal M Alowais (iD ${ }^{4}$ \\ 'Executive Directorate of Medical Affairs, \\ Saudi Red Crescent Authority, Riyadh, \\ Saudi Arabia; ${ }^{2}$ Department of Accident \\ and Trauma, Prince Sultan Bin Abdulaziz \\ College for Emergency Medical Services, \\ King Saud University, Riyadh, Saudi \\ Arabia; ${ }^{3}$ Department of Emergency \\ Medicine, Unaizah College of Medicine \\ and Medical Sciences, Qassim University, \\ Qassim, Saudi Arabia; ${ }^{4}$ Department of \\ Surgery, College of Medicine, Imam \\ Mohammad Ibn Saud Islamic University, \\ Riyadh, Saudi Arabia
}

Correspondence: Yousef M Alsofayan Executive Directorate of Medical Affairs, Saudi Red Crescent Authority,

P.O. Box: III29, Riyadh, III29, Saudi Arabia

Tel +966 1997।

Fax $+966 \quad$ II 2806666

Email y-m-alsofayan@hotmail.com
Purpose: The quality of resuscitation for out hospital cardiac arrest (OHCA) during the COVID-19 era could be affected. We aim to describe prehospital healthcare providers' resuscitative efforts for OHCA cases and their definitive outcomes.

Patients and Methods: This retrospective cross-sectional study included all OHCA cases between April and June 2021 across all regions in the Kingdom of Saudi Arabia (KSA). Demographic variables, response times, CPR providers, initial rhythm, use of AED/ Defibrillator, medical interventions, ROSC data, and dispatch codes were extracted from a central electronic platform.

Results: A total of 1307 OHCA cases were included in this study, males constituted $65 \%$ and $42 \%$ were $\geq 65$ years. Although the median response time to initiate CPR was 13 $\min , 11 \%$ of OHCA cases had a response time between 0 and $6 \mathrm{~min}$. About $75 \%$ of CPR was provided on scene by BLS units, $78 \%$ of OHCA cases had asystole as their initial rhythm, an AED/Defibrillator was used more than $90 \%$ of the time for pulseless VT/VF rhythm, and ROSC was achieved in $8 \%$ of OHCA patients.

Conclusion: During the COVID-19 pandemic, maintaining resuscitative efforts for OHCA continues in KSA. Closing knowledge gaps in the community and a better description of OHCA for the dispatcher could guide dispatch-assisted CPR and minimize OHCA response times.

Keywords: out of hospital cardiac arrest, CPR, ROSC, coronavirus disease, COVID-19

\section{Introduction}

In light of the Coronavirus Disease 2019 (COVID-19) pandemic, efforts to maintain essential healthcare services are been pursued globally. ${ }^{1}$ Priorities vary from one health system to another but all share the importance of resuscitations services for cardiac arrest cases. The challenge would go further to provide these kinds of services in the out of hospital settings. Interconnected communities, emergency medical services, and hospitals play a key role in the survival of the out of hospital cardiac arrest (OHCA). The use of an Automated External Defibrillator (AED) by a first responder on time and before the arrival of EMS providers on the scene, shorter response times intervals, and better quality of CPR is associated with improved outcomes. ${ }^{2}$ Recent data confirmed the importance of achieving ROSC in the field to predict survival for OHCA cases. ${ }^{3}$ On the other hand, the uncertainty of COVID-19 transmission for rescuers and EMS providers during resuscitative activities such as chest compression and the use of defibrillator could affect the quality and eventually the patient's survival rates. ${ }^{4}$ In the region, studying the burden of OHCA and its associated demographic characteristics will guide 
resuscitative strategies and minimize mortality rates. ${ }^{5}$ To curb the incidence of OHCA there is an urgent need to increase the public's exposure to basic life support educational programs and adapt targeted awareness campaigns. ${ }^{6}$

In this study, we aim to describe prehospital healthcare providers' resuscitative efforts for OHCA cases including demographic characteristics, response time to initiate CPR, interventions, and definitive outcomes in the Kingdom of Saudi Arabia (KSA) during the COVID-19 era.

\section{Materials and Methods Study Design and Setting}

This retrospective cross-sectional study included 1307 patients with OHCA, between April and June 2021 across all regions in KSA. All OHCA cases were included and there were no exclusion criteria.

\section{Data Collection}

OHCA demographic variables, response times, CPR providers, initial rhythm, use of $\mathrm{AED} /$ Defibrillator, airway interventions, vascular access, use of epinephrine, ROSC data, and dispatch codes were extracted from a central electronic platform of the Saudi Red Crescent Authority (SRCA) by two trained healthcare providers and filled into a separate excel sheet. Any discrepancies were modified by a third reviewer. Throughout the study, data privacy was maintained by restricted system access through unique passwords granted for data collectors after signing nondisclosure agreement forms.

\section{Study Variables}

OHCA was defined as any cardiopulmonary arrest with the cessation of cardiac mechanical activity that occurs outside of the hospital setting and is confirmed by the absence of signs of circulation. ${ }^{7}$ Ages were categories based on Index Mundi Classifications (in years): 0-14, 15-24, 2554, 55-64, and $\geq 65 .{ }^{8}$ Basic Life Support (BLS) Provider is a healthcare provider with a Basic Emergency Medical Technician Certificate and limited privileges: basic airway interventions (nasopharyngeal airway, oropharyngeal airway (OPA), bag-valve-mask ventilation (BVM)), chest compression, and AED operation. Advanced Life Support (ALS) Provider is a physician or a paramedic with Advanced privileges: Advanced airway interventions (endotracheal intubation), chest compression, use of a defibrillator, injection of epinephrine $1 \mathrm{mg} / 10 \mathrm{~mL}$ $(1: 10,000)$ solution. The local EMS system protocol dispatches either a BLS unit, an ALS unit, or both for all OHCA cases according to resources available. Initial rhythm was classified into Asystole, Pulseless Electrical Activity (PEA), and Pulseless Ventricular Tachycardia/ Ventricular Fibrillation (Pulseless VT/VF). Response time was defined as the time from the dispatcher receiving the OHCA call to the initiation of CPR at the scene. We classified EMS response times (in minutes) to 0-6, 7-9, $10-15,>15$ following a recent study by Johan Holmén et al. ${ }^{9}$ A local SRCA dispatch coding system uses numerical codes to identify certain initial caller's complaints as seen in the Supplemental Appendix and triage them accordingly.

\section{Study Outcomes}

The primary outcome of the study was the Return of Spontaneous Circulation (ROSC) in OHCA cases. Secondary outcomes include the use of an Automated External Defibrillator (AED)/Defibrillator in prehospital settings, response times to initiate CPR, various medical interventions utilized for OHCA cases, and initial dispatch codes to define OHCA probable causes.

\section{Ethical Considerations}

This study was approved by the Institutional Review Board on July 06, 2021, King Fahad Medical City, Riyadh, Saudi Arabia (Log Number: 21-284E).

\section{Statistical Analysis}

Descriptive statistics were used to describe categorical variables presented by counts and percentages. All percentages were rounded to one decimal. Continuous variables were analyzed based on the median and interquartile range (IQR). The analysis was done using Statistical Package for the Social Sciences 24 (SPSS-24).

\section{Results}

A total of 1307 OHCA cases were included in this study, males constituted $65 \%(n=849$ of 1307) of them. The nationality of the sample showed that $71 \%(n=924$ of 1307) of the cases were Saudis. Age categories differed among cases with the highest been $42 \%$ ( $n=544$ of 1307) for ages $\geq 65$ years, followed by $25-54$ years with $33 \%$ ( $n=436$ of 1307). Moreover, regions with the largest number of OHCA case were Riyadh 21\% ( $\mathrm{n}=276$ of 1307), Makkah 21\% ( $\mathrm{n}=274$ of 1307), and Eastern Province 19\% $(\mathrm{n}=249$ of 1307) as shown in Table 1. 
Table I Demographic Data of OHCA Cases During the Study Period

\begin{tabular}{|l|c|c|}
\hline Variable & Count & $\%$ \\
\hline Gender & 1307 & $100 \%$ \\
Male & 849 & $65 \%$ \\
Female & 458 & $35 \%$ \\
\hline Nationality & 1307 & $100 \%$ \\
Saudi & 924 & $71 \%$ \\
Non-Saudi & 383 & $29 \%$ \\
\hline Age categories (in years) & 1307 & $100 \%$ \\
0-I4 & 67 & $5 \%$ \\
I5-24 & 53 & $4 \%$ \\
$25-54$ & 436 & $33 \%$ \\
$55-64$ & 207 & $16 \%$ \\
$\geq 65$ & 544 & $42 \%$ \\
\hline Regions of OHCA & 1307 & $100 \%$ \\
Riyadh & 276 & $21 \%$ \\
Makkah & 274 & $21 \%$ \\
Madinah & 86 & $7 \%$ \\
Eastern Province & 249 & $19 \%$ \\
Qassim & 69 & $5 \%$ \\
Hail & 41 & $3 \%$ \\
Tabuk & 41 & $3 \%$ \\
Al Jawf & 35 & $3 \%$ \\
Northern Borders & 15 & $1 \%$ \\
Al Baha & 15 & $1 \%$ \\
Asser & 110 & $7 \%$ \\
Najran & 85 & \\
Jazan & & 11 \\
\hline
\end{tabular}

The median and (IQR) response time (in minutes) among all OHCA cases was 13 (9). $11 \%$ ( $\mathrm{n}=144$ of 1307) of OHCA cases had a response time (in minutes) between $0-6 \mathrm{~min}, 17 \%(\mathrm{n}=220$ of 1307$)$ between $7-9$ $\min , 38 \%(\mathrm{n}=501$ of 1307$)$ between $10-15 \mathrm{~min}$, and $34 \%$ $(n=442$ of 1307) $>15$ min. Further classifications of response times (in minutes) according to different regions in Saudi Arabia are illustrated in Figure 1.

$75 \%$ of the OHCA cases received CPR by BLS units $(\mathrm{n}=978$ of 1307$)$ and $25 \%$ of the cases through ALS units $(n=329$ of 1307). $78 \%(n=162$ of 208) of OHCA cases had asystole as their initial rhythm, whereas $12 \%$ ( $\mathrm{n}=26$ of 208) had PEA, and $10 \%(\mathrm{n}=20$ of 208) showed pulseless VT/VF. Prehospital care providers used the AED/ Defibrillator in $48 \%$ ( $n=627$ of 1307) among OHCA patients. A wide variety of airway interventions were utilized during OHCA resuscitation. OPA+BVM was used in $75 \%(\mathrm{n}=977$ of 1307$)$ cases, followed by BVM only in
$12 \%(\mathrm{n}=152$ of 1307$)$, and other less frequent airway interventions are shown in Table 2.

The most common vascular access used during OHCA was IV access in $41 \%(\mathrm{n}=533$ of 1307) of patients. Epinephrine was used in $6 \%(\mathrm{n}=84$ of 1307) and ROSC was seen in $8 \%(n=102$ of 1307) of all OHCA cases as shown in Table 2. 56\% of the ROSC cases were male $(\mathrm{n}=57$ of 102), their age (in years) median and (IQR) was 60 (35), their response time (in minutes) median and (IQR) was 13 (9), and the use of AED/Defibrillator was seen in $56 \%(n=57$ of 102$)$ of all ROSC cases.

Most frequent initial dispatch codes and their related response times (in minutes) median and (IQR) for OHCA were loss of consciousness (otherwise not specified) $32 \%$ $(\mathrm{n}=416$ of 1307) and a response time (in minutes) with a median and (IQR) of 12 (8), cardiopulmonary arrest $22 \%$ $(n=288$ of 1307), and a response time (in minutes) with a median and (IQR) of 11 (7), followed by respiratory illness and trauma $10 \%(\mathrm{n}=128$ of 1307) for each and response times (in minutes) with a median and (IQR) of 15 (8.25) and 13 (11), respectively, as seen in Table 3, Figure 2.

\section{Discussion}

To our knowledge, this is one of the earliest OHCA studies during the COVID-19 era in the region. In the past year, the ongoing COVID-19 pandemic has been linked to the increased incidence of OHCA. ${ }^{10}$ Globally, the pandemic had crippled healthcare systems in maintaining essential healthcare services and overwhelming available resources. ${ }^{1}$ National and international efforts in prehospital settings continue to identify clinical characteristics, risk factors, modes of transmission, and outcomes of COVID-19 to curb the spread of the disease in the communities. ${ }^{11-16}$ In KSA, efforts were taken to contain the pandemic through contact tracing, implementing curfew measures, and suspending domestic and international flights in early phases. ${ }^{14}$ Moreover, the surge capacity of hospitals was increased by $50 \%$ and the demand for prehospital services was evident. ${ }^{14}$ In addition, ambulances were to follow a strict local infection control protocol to prevent the spread of the disease among prehospital healthcare personnel and transported patients. Collectively, this affected the efficiency and response times to OHCA during the COVID-19 era. ${ }^{14}$

A recent scoping review of the OHCA in the gulf region showed a variety of demographic data, low CPR 


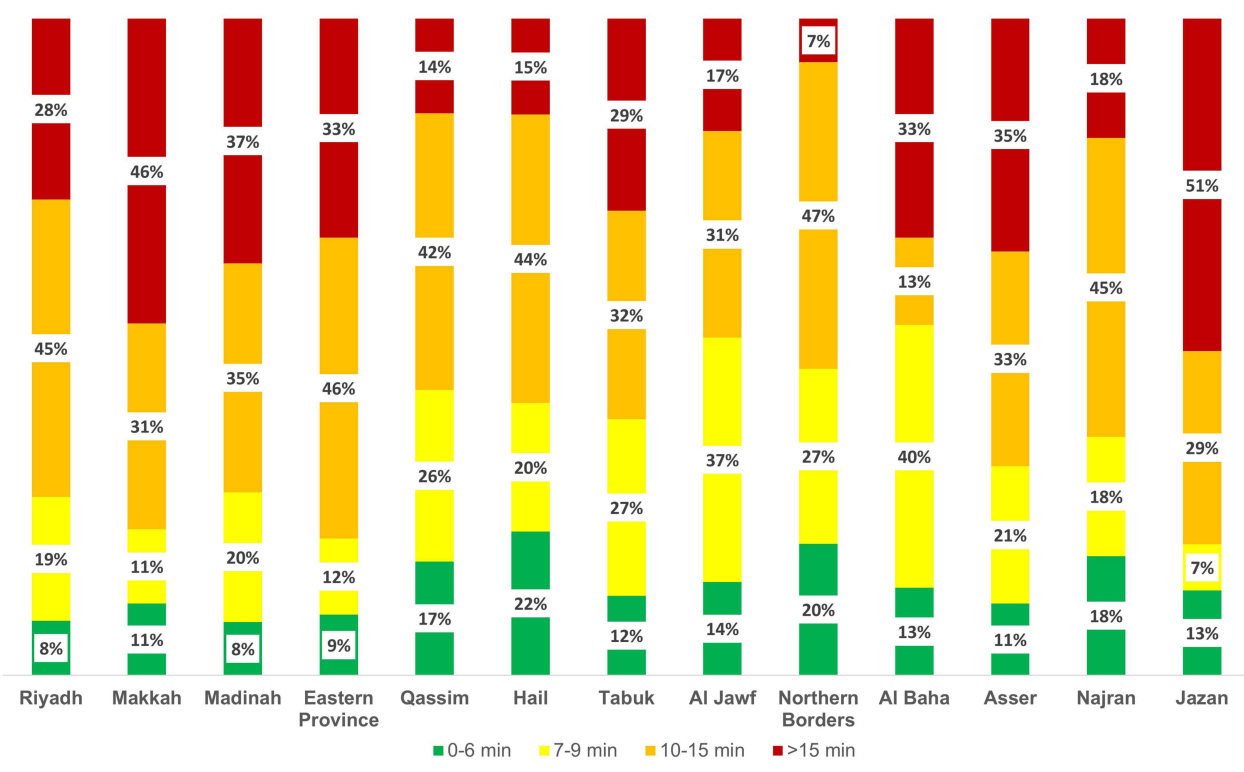

Figure I OHCA response times (in minutes) across different regions in Saudi Arabia during the study period.

rates, and fewer ROSCs in the gulf region compared with several western-based studies. ${ }^{17}$ In our study, we included a total of 1307 OHCA between April and June 2021. Not a surprise, as $65 \%$ of our sample were males, and that was similar to both local and international figures. ${ }^{7,18}$ Saudi represented $71 \%$ of all OHCA cases and this could be attributed to that $62 \%$ of the population in KSA are Saudis. ${ }^{19}$

Also, the highest age category in our study was $\geq 65$ years representing $42 \%$ of the sample. Again, these results show no difference when compared to a US OHCA surveillance registry and a local university hospital study in central KSA emphasizing the importance and special attention to geriatric OHCA. ${ }^{7,18}$ The distribution of OHCA cases across KSA regions was obvious with Riyadh and Makkah holding the highest numbers of $21 \%$ cases for each as this could be explained by the population growth in these regions. ${ }^{20}$ It has been proven that response time is an independent factor for predicting OHCA survival and even favorable neurological outcomes. ${ }^{9}$ Additionally, a recent study concluded that an optimal response time of $6.2 \mathrm{~min}$ is associated with survival to hospital discharge for OHCA. ${ }^{21}$ On a large scale, a recent meta-analysis of 24 studies showed that ambulance response times were higher during COVID19 when compared to the non-pandemic period $10.1 \mathrm{~min}$ and 9 min, respectively. ${ }^{22}$ Having said that, our response time varied depending on the availability of resources promptly with a median of 13 minutes across all regions. We noticed as well that $11 \%$ of OHCA cases received care between $0-6 \mathrm{~min}$ and $17 \%$ between $7-9 \mathrm{~min}$. Although these percentages are not far from previous studies, monitoring this variable and dissecting its related factors is crucial in improving response strategies to OHCA. ${ }^{9}$ Providing bystander CPR with or without the guidance of the dispatcher before the arrival of healthcare providers to the scene of an OHCA was associated with double the survival than not initiating CPR. ${ }^{23,24}$ However, during the COVID-19 era, this could pose a risk for the transmission of the disease for the bystanders, especially without personal protective equipment. ${ }^{4}$

Of notice, most OHCA resuscitation and interventions in our study were initiated by BLS units $75 \%$ of the time. Although Local protocols advise for ALS units in the scene for any OHCA case, the number of BLS units are higher in number and have easy access to various sites efficiently, hence their higher percentages of initially evaluating OHCA patients. The Initial rhythm was asystole in $78 \%$ of OHCA cases, $12 \%$ had PEA, 10\% showed pulseless VT/VF, and an AED/Defibrillator was used in more than $90 \%$ of the time for that rhythm. In OHCA, many studies during COVID-19 and non-pandemic period showed that unshockable rhythms are common and that those with shockable rhythms (pulseless VT/VF) are linked with better outcomes. ${ }^{7,18,22,24-26}$

A variety of airway interventions were seen among OHCA cases with OPA+BVM in $75 \%$ of the cases and ETT in $2 \%$. Knowing that survival has been linked in previous studies with no advanced airway interventions, utilizing supraglottic airway devices during pandemics 
Table 2 Type of CPR Provider, Initial Rhythm, Interventions, and ROSC of OHCA Cases During the Study Period

\begin{tabular}{|c|c|c|}
\hline Variable & Count & $\%$ \\
\hline EMS unit & 1307 & $100 \%$ \\
\hline BLS* & 978 & $75 \%$ \\
\hline $\mathrm{ALS}^{\times}$ & 329 & $25 \%$ \\
\hline Initial rhythm & 208 & $100 \%$ \\
\hline Asystole & 162 & $78 \%$ \\
\hline PEA & 26 & $12 \%$ \\
\hline Pulseless VT/VF & 20 & $10 \%$ \\
\hline AED/Defibrillator use & 1307 & $100 \%$ \\
\hline Yes & 627 & $48 \%$ \\
\hline Airway interventions & 1307 & $100 \%$ \\
\hline $\mathrm{NPA}^{\dagger}$ & 2 & $0 \%$ \\
\hline $\mathrm{OPA}^{\ddagger}$ & 70 & $5 \%$ \\
\hline $\mathrm{BVM}^{\top}$ & 152 & $12 \%$ \\
\hline $\mathrm{OPA}+\mathrm{BVM}$ & 977 & $75 \%$ \\
\hline $\mathrm{NPA}+\mathrm{BVM}$ & 2 & $0 \%$ \\
\hline ETT & 31 & $2 \%$ \\
\hline No airway intervention & 73 & $6 \%$ \\
\hline Vascular access & 1307 & $100 \%$ \\
\hline IV & 533 & $41 \%$ \\
\hline 10 & 8 & $0 \%$ \\
\hline No access & 766 & $59 \%$ \\
\hline Epinephrine use & 1307 & $100 \%$ \\
\hline Yes & 84 & $6 \%$ \\
\hline ROSC & 1307 & $100 \%$ \\
\hline Yes & 102 & $8 \%$ \\
\hline
\end{tabular}

Notes: *Basic life support provider. ${ }^{\times}$Advanced life support provider. ${ }^{\dagger}$ Nasopharyngeal airway. ${ }^{\ddagger}$ Oropharyngeal airway. "Bag-valve-mask ventilation.

seems a rationale option to minimize the risk of disease transmission. ${ }^{22,27}$

The highest first attempt success and most rapid in time access in OHCA patients is tibial intraosseous access. ${ }^{28}$ Due to the rapid transportation to hospital strategy, 59\% of OHCA cases had no access in the field. On the other hand, a causal relationship between OHCA survival and epinephrine dose could not be established. ${ }^{29} 6 \%$ of the patients in our study received epinephrine as the majority of the cases were managed by BLS units with limited privileges.

During the COVID-19 pandemic, a recent metaanalysis confirmed that the chances of ROSC for OHCA cases were lower at $18 \%$ when compared with the nonpandemic period of $21 \% .^{22}$ In this study, ROSC was seen in only $8 \%$ of OHCA cases and this could be related to the small sample of cases. $56 \%$ of the studied population were
Table 3 Response Times for OHCA Cases (in Minutes), Median (Interquartile Range) According to Dispatch Codes

\begin{tabular}{|l|c|}
\hline Dispatch Code & $\begin{array}{c}\text { Response Time (min) } \\
\text { Median (IQR) }\end{array}$ \\
\hline Total & $13(9)$ \\
Chest pain & $14(8)$ \\
Hypoglycemic attack & $14(6.75)$ \\
Communicable disease & $16(9)$ \\
Non-specific complain & $15(10)$ \\
Trauma & $13(11)$ \\
Respiratory illness & $15(8.25)$ \\
Others* & $13(9)$ \\
Cardiopulmonary arrest & $11(7)$ \\
Loss of consciousness (otherwise & $12(8)$ \\
not specified) & \\
\hline
\end{tabular}

Notes: *Others include: explosive hazard, fire, firearm use, electrical hazard, fall, detention, drowning, shocking, seizing episode, psychiatric illness, bleeding, childbirth, venomous poisoning, fight, heatstroke, suicidal attempt, use of a sharp object, and stroke dispatch codes.

male, with a median age of 60 years, a median response time of $13 \mathrm{~min}$, and $56 \%$ of them received $\mathrm{AED} /$ Defibrillator proving the importance of response times and initial shockable rhythm in ROSC.

OHCA cases had different initial dispatch codes and a variety of response times mapped according to the codes. Loss of consciousness (otherwise not specified) represented $32 \%$ with a median response time of $12 \mathrm{~min}$, cardiopulmonary arrest represented $22 \%$ with a median response time of $11 \mathrm{~min}$, followed by respiratory illness and trauma representing $10 \%$ each as shown in a previous study with a median response time of 15 and $13 \mathrm{~min}$, respectively. ${ }^{18}$ Only $3 \%$ of the initial dispatch codes for OHCA cases were communicable diseases with a response time of 16 min. Having said that, causes for OHCA during the COVID-19 pandemic varied but were less frequently linked to communicable diseases. The importance of public awareness regarding the caller's ability to identify and describe an OHCA case for the dispatcher is critical to be coded properly and guide dispatch-assisted CPR and minimize response times as evident in our results.

Therefore, we recommend maintaining a good resuscitation quality for OHCA patients during the COVID-19 era while maintaining personal protective equipment. Special attention should be addressed to the elderly and male gender with OHCA. A goal of 6 minutes response time should be maintained in the pre-hospital services, closing knowledge gaps in OHCA, improving bystander CPR policies as well as early use of AED, educating the 


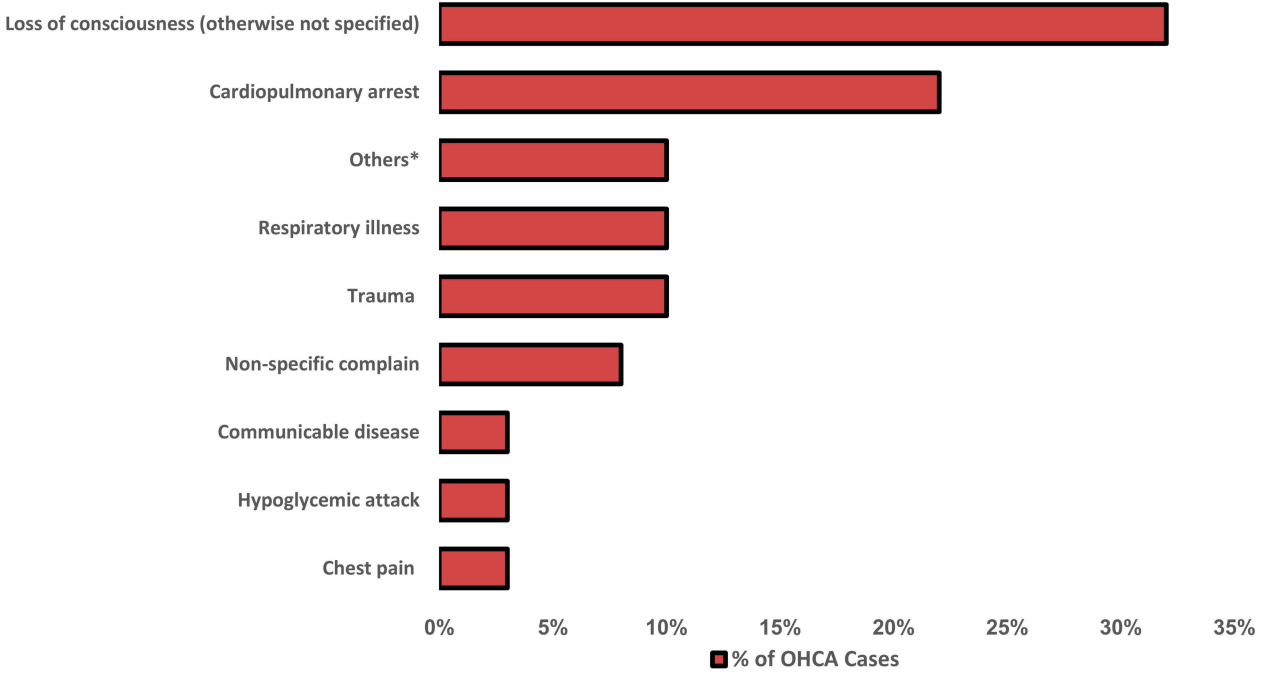

Figure 2 Percentage of OHCA cases according to initial dispatch codes during the study period. *Others include: explosive hazard, fire, firearm use, electrical hazard, fall, detention, drowning, shocking, seizing episode, psychiatric illness, bleeding, childbirth, venomous poisoning, fight, heatstroke, suicidal attempt, use of a sharp object, and stroke dispatch codes.

public to better describe an OHCA case to a dispatcher are factors linked with favorable outcomes. .,23,30-35 $^{-35}$

\section{Limitations}

This study has several limitations. Since the study design was retrospective cross-sectional, its weaknesses include the inability to reach causal inference of various factors with study outcomes. Also, many missing variables including OHCA patients' comorbidities, medications used during resuscitation, number of shocks delivered, and ROSC data with favorable neurological outcomes could have added to the value of the study. Finally, bystander CPR and 1st responder AED use were not captured, a factor that could further guide a national plan in combating OHCA. We suggest further prospective studies to address factors associated with favorable outcomes for $\mathrm{OHCA}$ in the region.

\section{Conclusion}

During the COVID-19 pandemic, maintaining resuscitative efforts for OHCA continues in KSA. The average time to initiate $\mathrm{CPR}$ for OHCA cases was 13 minutes and only $11 \%$ of them had a response time of 6 min and less. Most of the cases had a rhythm of asystole and ROSC was achieved in only $8 \%$. Closing knowledge gaps in the community and a better description of OHCA for the dispatcher could guide dispatch-assisted CPR and minimize OHCA response times.

\section{Data Sharing Statement}

Data of the participants are available upon reasonable request from the corresponding author.

\section{Acknowledgment}

We would like to thank the Medical Research Unit, Saudi Red Crescent Authority for providing the data required to achieve the results of this study.

\section{Author Contributions}

All authors made a significant contribution to the work reported, whether that is in the conception, study design, execution, acquisition of data, analysis and interpretation, or in all these areas; took part in drafting, revising or critically reviewing the article; gave final approval of the version to be published; have agreed on the journal to which the article has been submitted; and agree to be accountable for all aspects of the work.

\section{Funding}

No funding was provided for this manuscript.

\section{Disclosure}

All authors report no conflicts of interest for this work and certify that they have no affiliations with or involvement in any organization or entity with any financial interest or nonfinancial interest in the subject matter or materials discussed in this manuscript. 


\section{References}

1. World Health Organization. Maintaining essential health services: operational guidance for the COVID-19 context interim guidance. Available from: https://www.who.int/publications/i/item/WHO-2019nCoV-essential-health-services-2020.1. Accessed June 16, 2021.

2. McCarthy JJ, Carr B, Sasson C, et al. Out-of-hospital cardiac arrest resuscitation systems of care: a scientific statement from the American Heart Association. Circulation. 2018;137(21):e645-e660. doi:10.1161/CIR.0000000000000557

3. Sasson C, Rogers MAM, Dahl J, et al. Predictors of survival from out-of-hospital cardiac arrest: a systematic review and meta-analysis. Circ Cardiovasc Qual Outcomes. 2010;3(1):63-81. doi:10.1161/ CIRCOUTCOMES.109.889576

4. Couper K, Taylor-Phillips S, Grove A, et al. COVID-19 in cardiac arrest and infection risk to rescuers: a systematic review. Resuscitation. 2020;151:59-66. doi:10.1016/j.resuscitation.20 20.04.022

5. Batt AM, Al-Hajeri AS, Cummins FH. A profile of out-of-hospital cardiac arrests in Northern Emirates, United Arab Emirates. Saudi Med J. 2016;37(11):1206. doi:10.15537/smj.2016.11.16126

6. Subki AH, Mortada HH, Alsallum MS, et al. Basic life support knowledge among a nonmedical population in Jeddah, Saudi Arabia: Cross-Sectional Study. Interact J Med Res. 2018;7(2): e10428. doi: $10.2196 / 10428$

7. McNally B, Robb R, Mehta M, et al. Out-of-hospital cardiac arrest surveillance - Cardiac Arrest Registry to Enhance Survival (CARES), United States, October 1, 2005-December 31, 2010. MMWR Surveill Summ. 2011;60(8):1-19.

8. Index Mundi. Saudi Arabia age structure. Available from: https:// www.indexmundi.com/saudi_arabia/age_structure.html. Accessed June 8, 2021.

9. Holmén J, Herlitz J, Ricksten SE, et al. Shortening ambulance response time increases survival in out-of-hospital cardiac arrest. $J$ Am Heart Assoc. 2020;9(21):e017048. doi:10.1161/ JAHA. 120.017048

10. Baldi E, Sechi GM, Mare C, et al. Out-of-hospital cardiac arrest during the covid-19 outbreak in Italy. $N$ Engl $J$ Med. 2020;383 (5):496-498. doi:10.1056/NEJMc2010418

11. World Health Organization. Timeline: WHO's COVID-19 response. Available from: https://www.who.int/emergencies/diseases/novelcoronavirus-2019/interactive-timeline. Accessed June 26, 2021.

12. Alsofayan YM, Althunayyan SM, Khan AA, et al. Clinical characteristics of COVID-19 in Saudi Arabia: a National Retrospective Study. $J$ Infect Public Health. 2020;13(7):920-925. doi:10.1016/j. jiph.2020.05.026

13. Khan A, Althunayyan S, Alsofayan Y, et al. Risk factors associated with worse outcomes in COVID-19: a retrospective study in Saudi Arabia. East Mediterr Health J. 2020;26(11):1371-1380. doi:10.26719/emhj.20.130

14. Khan AA, Alsofayan YM, Alahmari AA, et al. COVID-19 in Saudi Arabia: the national health response. East Mediterr Health J. 2021. doi:10.26719/emhj.21.048

15. Khan AA, Alahdal HM, Alotaibi RM, et al. Controlling COVID-19 pandemic: a mass screening experience in Saudi Arabia. Front Public Health. 2021;8:1013. doi:10.3389/fpubh.2020.606385

16. Saudi Red Crescent Authority. Saudi red crescent authority. Available from: https://www.srca.org.sa/. Accessed June 23, 2021.

17. Batt A, Lanos C, Delport S, et al. Out-of-hospital cardiac arrests in the Gulf region: a scoping review. Eur J Emerg Med. 2020;27(1):e6. doi:10.1097/01.mej.0000697820.03632.98

18. Salleeh HM, Gabralla KA, Leggio WJ, et al. Out-of-hospital adult cardiac arrests in a university hospital in central Saudi Arabia. Saudi Med J. 2015;36(9):1071. doi:10.15537/smj.2015.9.12081
19. General Authority for Statistics. Population by gender, age groups and nationality (Saudi/Non-Saudi). Available from: https://www.stats. gov.sa/en/5680. Accessed July 5, 2021.

20. General Authority for Statistics. Population and housing census. Available from: https://www.stats.gov.sa/en/13. Accessed July 5, 2021.

21. Huang LH, Ho YN, Tsai MT, et al. Response time threshold for predicting outcomes of patients with out-of-hospital cardiac arrest. Emerg Med Int. 2021;11:2021. doi:10.1155/2021/5564885

22. Scquizzato T, D'Amico F, Rocchi M, et al. Impact of COVID-19 pandemic on out-of-hospital cardiac arrest system-of-care: a systematic review and meta-analysis. Prehosp Emerg Care. 2021:1-12. doi:10.1080/10903127.2021.1967535

23. Hasselqvist-Ax I, Riva G, Herlitz J, et al. Early cardiopulmonary resuscitation in out-of-hospital cardiac arrest. $N$ Engl $J$ Med. 2015;372(24):2307-2315. doi:10.1056/NEJMoa1405796

24. Gräsner JT, Herlitz J, Tjelmeland IB, et al. European resuscitation council guidelines 2021: epidemiology of cardiac arrest in Europe. Resuscitation. 2021;161:61-79. doi:10.1016/j.resuscitation.20 21.02.007

25. Atwood C, Eisenberg MS, Herlitz J, et al. Incidence of EMS-treated out-of-hospital cardiac arrest in Europe. Resuscitation. 2005;67 (1):75-80. doi:10.1016/j.resuscitation.2005.03.021

26. Conroy KM, Jolin SW. Cardiac arrest in Saudi Arabia: a 7-year experience in Riyadh. $J$ Emerg Med. 1999;17(4):617-623. doi:10.1016/S0736-4679(99)00049-9

27. McMullan J, Gerecht R, Bonomo J, et al. Airway management and out-of-hospital cardiac arrest outcome in the CARES registry. Resuscitation. 2014;85(5):617-622. doi:10.1016/j. resuscitation.2014.02.007

28. Reades R, Studnek JR, Vandeventer S, et al. Intraosseous versus intravenous vascular access during out-of-hospital cardiac arrest: a randomized controlled trial. Ann Emerg Med. 2011;58 (6):509-516. doi:10.1016/j.annemergmed.2011.07.020

29. Al-Mulhim MA, Alshahrani MS, Asonto LP, et al. Impact of epinephrine administration frequency in out-of-hospital cardiac arrest patients: a retrospective analysis in a tertiary hospital setting. $J$ Int Med Res. 2019;47(9):4272-4283. doi:10.1177/0300060 519860952

30. Qara FJ, Alsulimani LK, Fakeeh MM, et al. Knowledge of nonmedical individuals about cardiopulmonary resuscitation in case of cardiac arrest: a cross-sectional study in the population of Jeddah, Saudi Arabia. Emerg. Med. Int. 2019;2019:1-11. doi:10.1155/2019/ 3686202

31. Shams A, Raad M, Chams N, et al. Community involvement in out of hospital cardiac arrest: a cross-sectional study assessing cardiopulmonary resuscitation awareness and barriers among the Lebanese youth. Medicine. 2016;95(43):e5091. doi:10.1097/MD.0000000 000005091

32. Alsharari A, Alduraywish A, Al-Zarea E, et al. Current status of knowledge about cardiopulmonary resuscitation among the university students in the northern region of Saudi Arabia. Cardiol Res Pract. 2018;2018. doi:10.1155/2018/3687472

33. Al Enizi B, Saquib N, Zaghloul M, et al. Knowledge and attitudes about basic life support among secondary school teachers in Al-Qassim, Saudi Arabia. Int J Health Sci. 2016;10(3):415-422.

34. Alnajjar H, Hilal RM, Alharbi AJ, et al. Evaluation of awareness, knowledge, and attitudes towards basic life support among non-medical students at two academic institutions in Jeddah, Saudi Arabia. Adv Med Educ Pract. 2020;11:1015. doi:10.2147/AMEP. S271130

35. Public Access Defibrillation Trial Investigators. Public-access defibrillation and survival after out-of-hospital cardiac arrest. $N$ Engl $J$ Med. 2004;351(7):637-646. doi:10.1056/NEJMoa040566 


\section{Publish your work in this journal}

The Open Access Emergency Medicine is an international, peerreviewed, open access journal publishing original research, reports, editorials, reviews and commentaries on all aspects of emergency medicine. The manuscript management system is completely online and includes a very quick and fair peer-review system, which is all easy to use. Visit http://www.dovepress.com/testimonials.php to read real quotes from published authors.

Submit your manuscript here: https://www.dovepress.com/open-access-emergency-medicine-journal 